\title{
Alternative Energy Sources for Sensor Nodes: Rationalized Design for Long-Term Deployment
}

\author{
Alex S. Weddell, Nick R. Harris, and Neil M. White \\ School of Electronics and Computer Science, University of Southampton, SO17 1BJ, UK \\ +44 (0)23 80594996 \{asw05r, nrh, nmw\}@ecs.soton.ac.uk
}

\begin{abstract}
Energy harvesting is a means of extending the lifetimes of wireless sensor nodes. Here, we describe the current state-of-theart in energy harvesting technologies, and compare them against long-life primary batteries in terms of their total energy, economic cost and environmental impact. Issues affecting the lifetimes of energy harvesting devices, which are often overlooked, are described. We discuss the requirements for energy-awareness by wireless sensor network management algorithms, and how to deliver it for systems using batteries or energy harvesting devices and supercapacitors. A novel approach to monitoring state-of-charge, and an embedded software architecture for energy management (which has been deployed on battery-powered and energyharvesting nodes), are introduced. This new 'energy stack' structures the node's energy-related operations, while hiding their complexity from the application layer, and providing a straightforward interface. We present a complete approach to designing the energy-related aspects of a node for long-term deployment, including hardware choices and embedded software design.
\end{abstract}

Keywords - energy management, wireless sensor networks, energy harvesting

\section{INTRODUCTION}

$\mathrm{T}$ HE current state-of-the-art in commercial off-the-shelf energy harvesting technologies, for example in vibration energy harvesting and indoor photovoltaics, yield power levels of the order of a milliwatt in typical operating conditions [1], [2]. While these power levels may appear restrictively small, the operation of harvesting elements over a number of years can mean that the technologies are broadly comparable with long-life primary batteries, both in terms of energy provision and the cost per joule of energy provided. Moreover, systems incorporating energy harvesting will typically be capable of recharging after depletion - a feature that is lacking in systems powered by primary batteries.

There are other advantages of using energy harvesting, including the ability to monitor more closely the amount of energy being used by a system and hence deliver an improved level of energy-awareness, as may be required for state-ofthe-art sensor network management algorithms [3].

The work reported on in this paper was undertaken as part of the Data Information Fusion Defence Technology Centre (DIF DTC) Phase II 'Adaptive Energy-Aware Sensor Networks' project, funded jointly by the UK Ministry of Defence and General Dynamics UK.
Supercapacitors are often seen as ideal energy buffers that do not degrade over time. It may be true that they do not exhibit some of the less desirable characteristics of rechargeable batteries (such as memory effects and complex charging requirements), but supercapacitors do have a finite lifetime and must be operated in carefully controlled conditions in order to extend this.

The aim of this paper is to review and compare the capabilities and practical considerations of commercial energy-harvesting systems and long-life primary batteries. We also introduce methods for determining state-of-charge, and a flexible software stack that takes account of changing hardware without altering the program structure.

We conclude that energy-harvesting systems are broadly cost-comparable with systems using long-life primary batteries, over a ten-year lifetime. Energy-harvesting systems offer benefits of finer-grained energy awareness and the ability to recharge, but we find that in deployments where operating conditions are likely to be extreme, supercapacitor lifetimes are reduced considerably.

\section{TOTAL ENERGY OVER LIFETIME}

\section{A. Lifetime expectations}

In this section we compare the total energy gained from a long-life high-capacity cell, with that which can be expected from an energy-harvesting system over its lifetime. Lithium thionyl chloride $\left(\mathrm{LiSOCl}_{2}\right)$ is a commercially-available battery type that has been used in long-term wireless sensor deployments [4], with an expected shelf life of around ten years [5]. We compare it with the total energy that can be expected over ten years from an amorphous silicon photovoltaic (PV) cell in a typical office environment, and a vibration energy harvester on a piece of machinery. We discuss the long-term behavior of super-capacitors, often used as buffers storing harvested energy.

\section{B. Typical energy sources}

An indoor PV cell, such as a Schott Solar OEM module with dimensions $90 \times 72 \mathrm{~mm}$, can typically harvest around $0.80 \mathrm{~mW}$ at 500 lux (normal light levels for a well-lit office) [1]. It is reasonable to expect such a module to operate for more than ten years in these conditions without significant 
deterioration [6]. Assuming a 500 lux light level for 8 hours per day over 10 years, the total energy delivered by this device over 10 years is around $84 \mathrm{~kJ}$.

A Perpetuum PMG17-120 vibration energy harvester generates energy from "twice-line-frequency" machine vibration, and operates between $0.025 g$ and $1.0 \mathrm{~g}$ (where $g$ is a RMS acceleration of $9.81 \mathrm{~ms}^{-2}$ ). For vibration magnitudes at its minimum $0.025 g$, the device will provide a typical usable power output of $0.80 \mathrm{~mW}$ [2]. Assuming 24-hour operation over ten years, the device will provide around $252 \mathrm{~kJ}$.

A LiSOCl ${ }_{2}$ battery has a quoted 10 -year lifetime, with a ' $\mathrm{C}$ ' sized cell providing $8.5 \mathrm{Ah}$ at $3.6 \mathrm{~V}$ [5]. This equates to approximately $110 \mathrm{~kJ}$ of energy over its lifetime.

The three energy sources presented above, and investigated in more detail later in this report, are broadly comparable on the total energy they can deliver over a ten year period.

\section{Energy stores}

Whereas typical wireless sensor nodes can be powered directly from a $\mathrm{LiSOCl}_{2}$ cell (due to its stable $3.6 \mathrm{~V}$ output and ability to support currents up to $100 \mathrm{~mA}$ ), the same cannot be said for energy harvesting devices. Energy from a PV cell must be stored in order to sustain operation overnight, and energy harvesting devices generally do not provide sufficient instantaneous currents for the demands of wireless sensor nodes. Commercial off-the-shelf supercapacitors, such as the Panasonic Gold HW series, offer capacitances of several farads, can support discharge currents of hundreds of milliamps, and behave similarly to conventional aluminum electrolytics. Unfortunately, they also exhibit similar lifetime dynamics. For example, the $1 \mathrm{~F}$ version has a maximum operating voltage of $2.3 \mathrm{~V}$, maximum operating temperature of $70^{\circ} \mathrm{C}$, and guaranteed lifetime of 1,000 hours [7].

The lifetime extension due to operating temperature reduction can be expressed by the Arrhenius Equation, which indicates that the expected lifetime will double for every $10^{\circ} \mathrm{C}$ reduction in operating temperature [7]. Furthermore, investigations into large supercapacitor behavior indicate that the expected lifetime will also double for every $0.1 \mathrm{~V}$ operating voltage reduction [8]. Therefore, by operating the supercapacitor at room temperature and at $1.8 \mathrm{~V}$, it can be expected that its lifetime will be extended in excess of the desired 10-year lifetime. 'End of life' for a supercapacitor is normally the point at which its internal resistance has quadrupled and its capacitance has decreased by around $20 \%$. After this point, these parameters can be expected to deteriorate more quickly (there is a definite 'inflection point' in the supercapacitor parameters shortly after this time) [8].

The available capacity of a $\mathrm{LiSOCl}_{2}$ battery is dependent on temperature, with the capacity at $72^{\circ} \mathrm{C}$ being around half of that at $25^{\circ} \mathrm{C}$. The effect of temperature is less pronounced on long-life lithium batteries than supercapacitors, but extremes will clearly shorten their lifetime. They are also sensitive to discharge rate, but this is a complex relationship dependent on temperature [5].

\section{Cost OVER LifETIME}

\section{A. Energy harvesting - photovoltaics and vibration}

PV modules as described in II.B are available in volume at a cost of around $\$ 5$ per unit [9]. Supercapacitors, such as the Panasonic Gold 1F, are available at a cost of approximately $\$ 3$ in volume [10]. Assuming two supercapacitors are connected in series in each system (to maintain a low working voltage for lifetime extension purposes) a cost of under $\$ 15$ for the energy subsystem is achievable.

Vibration energy harvesting is an emerging technology, and vibration harvesters are expensive compared to PV modules. The PMG17 microgenerator is priced at around $\$ 700$ in volume [11], which is justified by the manufacturing effort required to produce the units, and the relatively small number of sales achieved by the complex technology thus far (the price can be expected to fall with increasing sales).

Vibration energy harvesters can achieve sustained power outputs of up to $40 \mathrm{~mW}$ if vibration is plentiful, delivering a theoretical maximum of $12.6 \mathrm{MJ}$ over ten years. This outperforms the quoted $\mathrm{LiSOCl}_{2}$ batteries by two orders of magnitude. Theoretically, the vibration energy harvester could save over 100 battery changes (costing around $\$ 1,500$ in parts, plus costs associated with labor and disposal).

\section{B. Lithium cell}

The previously discussed ' $\mathrm{C}$ ' size $\mathrm{LiSOCl}_{2}$ cells from Tadiran cost around $\$ 15$ in volume [10], and require no conditioning circuitry due to their stable operating voltage. It is difficult to monitor the state-of-charge of lithium cells with any accuracy (although it is possible to determine when the battery is discharged within the last $5 \%$ of its capacity) [5]. The question of energy awareness is addressed in further detail in Section V, but careful design is necessary to ensure that the energy requirement of the circuit does not exceed what the battery is able to provide over its projected lifetime.

The implications of battery replacement must be taken into account, especially where sensors are deployed in embedded or inaccessible locations. It is likely that labor costs will exceed the cost of the actual battery, added to the fact that node maintenance may disrupt the processes it is designed to monitor. In summary, designers must look at more than just the cost of the battery when comparing the economics of long-life lithium cells against energy-harvesting techniques.

\section{ENVIRONMENTAL CONSIDERATIONS}

\section{A. Lithium cells}

While $\mathrm{LiSOCl}_{2}$ cells do not contain lead, mercury or nickel like some other cells, they are nevertheless volatile and potentially toxic. They must not be incinerated due to risk of explosion, and abuse (physical, electrical, or thermal) of the cell can result in corrosive fluids leaking from the battery. The resulting vapors are irritants, and the inhalation of 
thionyl chloride can be extremely harmful. Furthermore, the contents of the cell react violently with water [12]. Recycling of lithium primary cells is in its infancy, but is possible through a number of processes. The requirement for controlled disposal of $\mathrm{LiSOCl}_{2}$ cells makes them unsuitable for 'deploy and forget' applications, and adds a substantial end-of-life systems cost. It should be noted that the thionyl chloride cathode presents the main hazard in $\mathrm{LiSOCl}_{2}$ cells.

\section{B. Energy harvesting devices and supercapacitors}

Panasonic recommend that their supercapacitors are disposed of by burning at a high temperature (lowtemperature burning results in production of toxic gases from the plastic sleeve). It is recommended that supercapacitors are crushed or pierced before incineration to reduce the risk of explosion. Panasonic Gold capacitors use an organic electrolyte and activated carbon electrodes. It is stated by the manufacturers that recycling is not required [7].

Amorphous silicon PV cells contain a small amount of silver, but in insufficient amounts to make its reclamation economically viable. They contain insufficient quantities of regulated materials for them to be classified as hazardous, so simple disposal is generally economic for these items [13].

Electromagnetic vibration energy harvesters are typically of a metallic construction, featuring neodymium iron boron $\left(\mathrm{Nd}_{2} \mathrm{Fe}_{14} \mathrm{~B}\right)$ magnets. While these magnets are sometimes classed as 'rare-earth' magnets, they are in relatively plentiful supply. There are few hazards associated with these devices, except for the effects of their strong magnetic field. Their value can justify recycling in larger volumes [14].

\section{ENERGY AWARENESS}

\section{A. Energy monitoring}

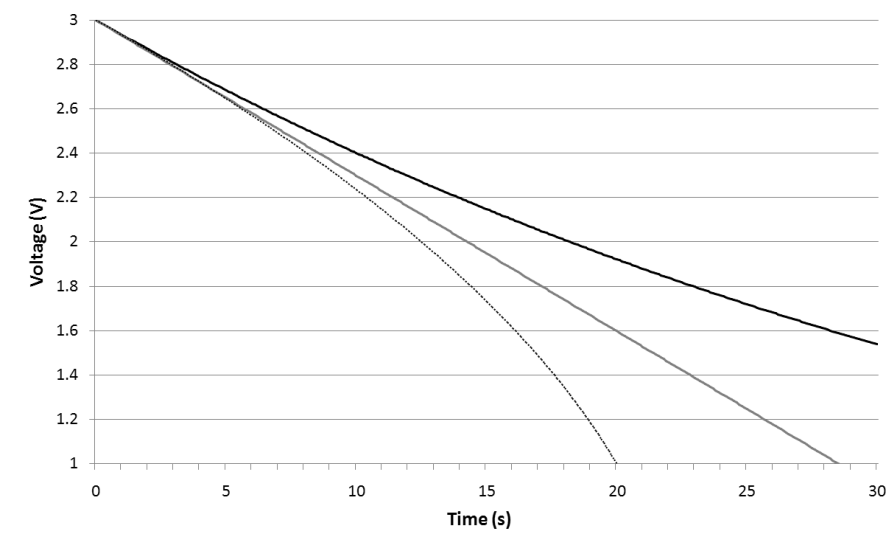

Fig. 1 Simulated voltage curves for a $1 \mathrm{~F}$ supercapacitor discharging through a constant resistance, constant current, and constant power load

It is important for energy-aware systems to have an indication of the state of charge of energy stores, in order that systems can adjust their behavior dependent on their energy status. There are a number of different ways of calculating this value, and these largely depend on the dynamics of the load discharging the energy store. Rather than simply an indication of how full the energy store is, it may be helpful to get an idea of how long a node can operate for compared to its neighbors. Fig. 1 shows that the load type has a major effect on the discharge characteristics, with the curves diverging substantially. The graph shows an example of the discharge profile of a $1 \mathrm{~F}$ supercapacitor through a $450 \Omega$ resistor, $70 \mathrm{~mA}$ current load, and $200 \mathrm{~mW}$ power load. These values were chosen as they draw similar power levels at $3 \mathrm{~V}$.

\section{B. State-of-charge determination for supercapacitors}

There will typically be a range of voltages over which a microcontroller can operate: this may, for example, be between 2.0 and 3.6 volts. Obviously, if such a microcontroller is driven directly from an energy store, the energy stored when the voltage is below 2.0 volts is effectively unusable. For a capacitor, the following methods for determining the energy status ( $t_{\text {frac}}$, the fraction of operating time remaining compared to when the store is full) are applicable, and largely rely on the relationship $E=\frac{1}{2} C V^{2}$ :

1. Energy fraction: this is simply a measure of how 'full' the energy store is, and is most useful when the load imposes a power requirement on the store. It is calculated by determining the usable energy stored, and dividing by the maximum usable energy if the store was full.

2. Voltage fraction: this value assumes that the load imposes a current requirement on the store. It is given by dividing the usable voltage range by the maximum usable voltage range.

3. Logarithmic discharge fraction: this value assumes a resistive load is present across the store. It gives an indication of the proportion of time remaining, expressed as a percentage (where $100 \%$ is fully charged, $0 \%$ is when the store is at the minimum voltage, and $50 \%$ is half way along the time axis between the two values) with the simplifying assumption that the capacitor is discharged through a constant resistance.

Clearly, a value for the energy proportion is not particularly helpful if the load across the store behaves as a currentdominated consumer or a resistance, as in these cases the power requirements of the node vary with the amount of energy stored (and hence the store voltage). The energy fraction is calculated by means of (1).

$$
t_{\text {frac }}=E_{\text {frac }}=\frac{E_{\text {present }}-E_{\text {min }}}{E_{\max }-E_{\min }}
$$

$E_{\text {present }}$ is the amount of energy stored by the system at a given time. $\mathrm{E}_{\min }$ is the energy at the store's minimum voltage, and $\mathrm{E}_{\max }$ is the energy at its maximum voltage. This gives a rough idea of the energy status of the node, but is reliant on the assumption that the load is a power-dominated consumer.

Our investigations show that, when exposed to varying voltages, a TI/Chipcon CC2431EM module behaves more 
closely as a current-dominant load than a resistive or powerdominant load. The voltage fraction gives an indication of the state-of-charge of the capacitor assuming it is discharged by a current which is independent of the store voltage or energy, and hence $I=C \frac{d V}{d T}$, used in (2).

$$
t_{\text {frac }}=V_{\text {frac }}=\frac{V_{\text {present }}-V_{\text {min }}}{V_{\max }-V_{\min }}
$$

$\mathrm{V}_{\text {present }}$ is the store voltage at a given time. $\mathrm{V}_{\min }$ and $\mathrm{V}_{\max }$ are the minimum and maximum store voltages.

Finally, the logarithmic discharge fraction exploits the fact that the voltage across a capacitor obeys the relationship $V=V_{0} e^{-t / C R}$ for a constant resistance and capacitance (where $\mathrm{V}_{0}$ is the initial voltage, $\mathrm{t}$ is time elapsed, and $\mathrm{C}$ and $\mathrm{R}$ are capacitance and resistance respectively). This can be rearranged to find $t$, as shown in (3).

$$
t=C R \ln \frac{V}{V_{0}}
$$

Thus to find the logarithmic discharge fraction, the result (4) is independent of $t, C$ and $R$.

$$
t_{\text {frac }}=1-\frac{\ln \frac{V}{V_{\max }}}{\ln \frac{V_{\min }}{V_{\max }}}
$$

Although this quantity is the most computationally expensive to calculate, it is likely to give a useful indication of charge status, as it assumes a resistive discharge of the energy store (i.e. the amount of energy consumed by the system is dependent on the store voltage, which is in turn dependent on the energy stored). This calculation can be implemented on microcontrollers using a Taylor expansion. Taking the expansion to its fourth term results in a typical error of approximately $3 \%$, and to its fourth term the error is around $1 \%$. This will normally be sufficiently accurate to give a good idea of the state-of-charge of the system.

\section{State-of-charge determination for primary batteries}

$\mathrm{LiSOCl}_{2}$ cells feature a stable operating voltage of around $3.6 \mathrm{~V}$ until they become depleted [5]. Hence, state-of-charge determination is non-trivial, and systems must estimate the amount of energy used to identify their energy status.

For more conventional primary cells, such as alkaline manganese dioxide batteries, the closed circuit voltage (CCV) of the cell must be measured to give an accurate idea of their state of charge. Measuring the open circuit voltage (OCV) of the cell will not give an accurate indication of the service life remaining. The $\mathrm{CCV}$ can be determined by placing the battery under a load for one or two seconds, and measuring its voltage. The load is dependent on battery size, but Energizer recommends a $10 \Omega$ load is used for a single $1.5 \mathrm{~V}$
AA-size cell [15]. The resultant $\mathrm{CCV}$ can then be traced across to the voltage profile of the cell, such as that given by the manufacturer [16], and mapped to straight lines through a piecewise-linear approximation (to simplify implementation in microcontrollers) as shown in Table 1.

TABLE I

Simplified Discharge PROFILE:

\begin{tabular}{c|rrr} 
DURACELL ALKALINE 'AA'-SIZE CELL THROUGH RESISTIVE LOAD \\
\hline Point & Voltage V & $\begin{array}{l}\text { Service } \\
\text { hours }\end{array}$ & $\begin{array}{l}\text { Remaining } \\
\text { lifetime }\end{array}$ \\
\hline 1 & 1.52 & 0 & $100 \%$ \\
2 & 1.37 & 15 & $86 \%$ \\
3 & 1.22 & 79 & $27 \%$ \\
\hline L & 1.00 & 108 & $0 \%$ \\
\hline 4 & 0.80 & 140 & $-29 \%$ \\
\hline
\end{tabular}

Any discharge curve can be approximated to a series of straight lines and stored in memory. The choice of curve will depend on the type of load normally attached to the battery. It must be noted that use of a resistive discharge curve does not imply that the microcontroller behaves as a constant resistance at all times: purely that its dynamic power consumption due to different voltages varies in line with that of a resistor.

The interrogation process is straightforward, but imposes a further energy requirement on the battery. For example, if the battery is tested once per hour for one year (and each test takes one second), with a $10 \Omega$ load, this will consume around $2.2 \%$ of the battery's total capacity. The system designer must trade off higher resolution on the battery state-of-charge against excessive energy wastage in testing.

\section{End-of-life identification for primary batteries}

End-of-life identification for $\mathrm{LiSOCl}_{2}$ batteries is possible. With a pulsed discharge, similar to that used to identify stateof-charge in alkaline batteries, end-of-life can be identified up to $15 \%$ before the cut-off voltage. Otherwise, continuous loading of the cell will only permit passive identification of end-of-life up to $3 \%$ before cut-off. The response of the cell can be sensitive to temperature variation, so care must be taken to ensure that any voltage drops due to pulsed discharges are not due to seasonal variations [5].

\section{E. Ageing}

Supercapacitors and secondary batteries exhibit 'ageing' effects, where parameters such as internal resistance and capacity degrade. In the case of supercapacitors, the decline in capacity has been found in previous studies to approximate to a straight line on an Arrhenius plot, with an inflection after its end-of-life. Given that electrolytic capacitors generally have a wide initial tolerance, and an ageing process that is dependent on temperature, humidity, and operating voltage [7], it is unlikely that the effects on capacity in a deployed system due to ageing will be accurately predicted. An approximation will likely suffice for the purposes of energyaware algorithms. 


\section{OTHER FACTORS}

\section{A. Lifetime of electronics}

Flash data retention is specified at a minimum of 100 years at $25^{\circ} \mathrm{C}$ for TI microcontrollers such as the MSP430. Flash lifetimes at higher temperatures can be predicted by using the Arrhenius equation. At $50^{\circ} \mathrm{C}$, memory retention can be expected to fall to 17 years [17]. Increased temperatures affect other parts of the system such as the energy store and harvesting device. Designers must consider the effects of the operating environment on all aspects of the system, to ensure that the required operating lifetime is achievable.

\section{B. Reliance on external maintenance}

PV cells are reliant on sufficient levels of light penetrating the glass to reach the $\mathrm{p}-\mathrm{n}$ junction. Over a 10 year deployment, significant residue can be expected to build up on a sensor node package, especially in an industrial environment. This can significantly degrade the cell performance, and hence reduce the harvesting efficiency. It must be ensured that the sensor package can be easily cleaned, or that the effects of dust and residue are minimized.

For battery powered sensors, the mechanics and impact of battery replacement must be considered. Furthermore, the maintenance of sensors should be carried out in a timely manner and a means for sensors to identify and communicate their low-energy status must be provided.

\section{SOFTWARE Structure FOR ENERGy MANAGEMENT}

\section{A. Justification}

We have detailed the complexities of energy management in wireless sensor nodes, and described why knowledge about the energy status of nodes is important. Standard operations such as state-of-charge determination can be complex, and we have outlined a number of methods for state-of-charge determination and end-of-life identification in primary cells and supercapacitors. This report has also described the ageing effects on supercapacitors and the impact that these have on an energy-harvesting system.

Energy management is a complex task, but is frequently overlooked in favor of communications algorithm development. We would argue that energy management should be treated with equal importance to communications. We believe that there are synergies between communications subsystems and energy management subsystems, in that algorithms are needed to manage physical hardware, process information from it, and present it to the application running on a sensor node. It is for this reason that we have developed an energy management stack, which exists alongside the communication stack in the node's embedded software [18].

The energy stack splits the energy management operations into three distinct layers, each layer interfacing with the layer directly below it and hiding its complexities from the layer above. This structure, and the provision of a standardized interface to the application running on the node, promotes modularization and code re-use, while delivering systems that can efficiently manage their energy resources.

\section{B. Software structure}

A consistent solution has been developed, in which the energy management software tasks are modularized and arranged into an energy stack. The energy management process is divided into three layers:

- Physical Energy Layer (PYE): obtains information about voltages of energy stores, controls physical switching, and manages energy converters to direct the energy flow.

- Energy Analysis Layer (EAN): takes data from the PYE and (through use of models of energy sources and stores) calculates the amount of energy stored.

- Energy Control Layer (ECO): takes a high-level view of the energy subsystem - controlling the transfer of charge between energy sources and stores if necessary. It reports on the overall energy status of the node, in a format which is independent of the node's energy hardware.

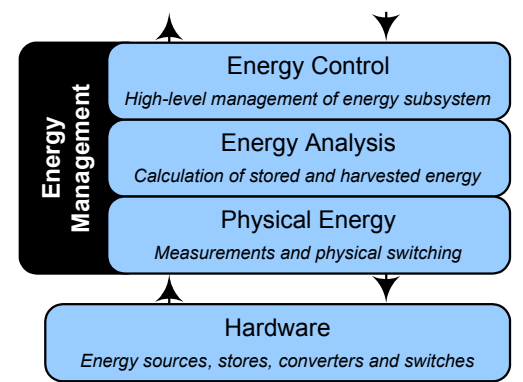

Fig. 2. The 'energy stack' software structure

The stack has access to an 'energy hardware information table', which gives details of the characteristics of the energy sources and stores, and the connections between them. This also defines which $\mathrm{I} / \mathrm{O}$ pins of the energy controller are connected to energy switches, the control pins of energy converters, or the outputs of sources or stores (for the purposes of voltage monitoring). Fig. 2 shows the layout of the energy stack. The stack can be queried by the application layer to determine the overall energy status of the node, and it is expected that it will be called at regular intervals so that it can carry out tasks and detect trends in the energy subsystem.

\section{Interface with the application layer}

At present, the ECO layer presents the application layer with an interface allowing it to see the important aspects of the energy status of the node. This includes the Power Priority value [3], an estimate of the usable energy stored, and a value for the supply voltage. The interface also permits the application layer to alter the threshold values for power priorities, and to prompt the energy stack to refresh by taking new readings. Future enhancements are planned to allow the application layer to monitor the instantaneous power delivered by energy sources. Further, more technical 
operations are hidden from the application layer, and implemented by functions further down the stack. For example, functions to calculate the state-of-charge of the energy store are implemented in the EAN layer.

\section{Prototype 1: System powered by primary batteries}

The discharge curve for a standard Duracell 'AA'-sized alkaline manganese dioxide cell has been adapted from the manufacturer's handbook. We have approximated the discharge curve to three straight lines (described by 4 points), as shown in Table 1. Point $\mathrm{L}$ has been found through interpolation, and is the lowest voltage at which the microcontroller can function, assuming it is powered by two cells in series (thus point L provides a $2.0 \mathrm{~V}$ supply). The data in this table is designed to be used in conjunction with a short low-impedance test as described in Section V.C. The remaining lifetime percentage is derived from the effective number of service hours expended and number of service hours available from the cell. To a large extent, the actual load that the cell normally drives is irrelevant: this test is used purely to obtain a value for state-of-charge.

In the energy stack, the PYE layer deals with switching a transistor in order that the cell can be discharged through a $10 \Omega$ load. It also measures the voltage at the end of the test. The EAN layer uses the data from Table 1 along with the voltage at the end of the test to estimate the remaining lifetime percentage. This is then communicated to the ECO layer which translates this data into energy priorities and levels for presentation to the application layer.

\section{E. Prototype 2: System powered by photovoltaic cell}

In this prototype, a PV cell provides the power for the system. Its input is passed through a switching converter and energy is stored in a $0.5 \mathrm{~F}$ supercapacitor. In this case, the PYE layer monitors the voltage across the supercapacitors. The EAN layer calculates the voltage fraction value, as shown in (2). This value is then interpreted by the ECO layer for presentation to the application layer, in the same format as in the first prototype. Due to the flexible design of the energy stack, minimal changes are needed to represent the change in energy source (the voltage fraction is calculated rather than the battery state-of-charge, and the battery model is ignored in favor of the capacitor equation).

\section{CONCLUSIONS}

We have explored the capabilities of energy harvesting devices and long-life $\mathrm{LiSOCl}_{2}$ batteries, and assessed their performance over a ten-year period. We have found that energy harvesting technology is broadly cost-comparable with long life batteries, and can potentially deliver longer system lifetimes. Assessing the state of charge of $\mathrm{LiSOCl}_{2}$ batteries is non-trivial until the end of their lifetime, and it is relatively straightforward to assess the state of charge of supercapacitors. We have described three main ways of expressing the state of charge of a supercapacitor, and explained that the type of discharge dictates the best method.
Algorithms for determining these quantities, along with the state-of-charge of primary batteries, have been presented and are suitable for implementing on a resource-constrained microcontroller. These methods form part of a system-wide approach to energy-awareness, including an 'energy stack' that has been implemented. The 'energy stack' has been demonstrated to present a flexible method of structuring a node's energy-related operations, while hiding their complexity from the application layer and providing it with a straightforward interface.

We have found that energy harvesting systems are best suited to energy-aware routing and management algorithms which monitor stored energy and adjust the node workload accordingly. Other factors which must be considered when designing nodes for long deployments have also been explored.

\section{REFERENCES}

[1] ASI ${ }^{\circledR}$-OEM Solarmodules for Indoor, Schott Solar GmbH, Putzbrunn, Germany, 2006.

[2] Perpetuum PMG17-120 Product Specification, Perpetuum Ltd, Southampton, UK, 2007.

[3] G. V. Merrett, N. R. Harris, B. M. Al-Hashimi, and N. M. White, "Energy Managed Reporting for Wireless Sensor Networks," Sensors and Actuators A: Physical. In press.

[4] K. Martinez, R. Ong, and J. Hart, "Glacsweb: a sensor network for hostile environments," in Proc. 1st IEEE Comm. Soc. Conf. on Senor and Ad Hoc Comms and Networks, 2004, pp. 81-7.

[5] Lithium Batteries Technical Brochure, Tadiran Batteries GmbH, Büdingen, Germany, 2006.

[6] D.L. King, J.A. Kratochvil, and W.E. Boyson, "Stabilization and performance characteristics of commercial amorphous-silicon PV modules," in Conf. Rec. 28th IEEE PV Spec.Conf., Anchorage, AK, 2000.

[7] Gold Capacitor Technical Guide, Panasonic Industrial Company, Secaucus, NJ, 2005.

[8] T. Umemura, Y. Mizutani, T. Okamoto, T. Taguchi, K. Nakajima, and K. Tanaka, "Life expectancy and degradation behavior of electric double layer capacitor Part I," in Proc. IEEE Int. Conf. on Prop. and App. Of Dielectric Materials, 2003, pp 944-948.

[9] Price List for Original Equipment Manufacturer (detailed), Schott Solar GmbH, Putzbrunn, Germany, 2006.

[10] Farnell UK, Products [Online], accessed 1 November 2007. Available: http://uk.farnell.com/

[11] Perpetuum Ltd., Products [Online], accessed 1 November 2007. Available: http://www.perpetuum.co.uk/home.php?page id=6

[12] Guidelines for Disposal of Lithium Cells and Batteries, Tadiran Batteries $\mathrm{GmbH}$, Büdingen, Germany.

[13] C. Eberspacher, and V. M. Fthenakis, "Disposal and recycling of endof-life PV modules," in Proc. 26th IEEE Photovoltaic Spec. Conf., Anaheim, CA, 1997, pp.1067-1072.

[14] A. Saguchi, K. Asabe, T. Fukuda, W. Takahashi, and R. O. Suzuki, "Recycling of rare earth magnet scraps: Carbon and oxygen removal from Nd magnet scraps," J. Alloys Compounds, v 408-412, Feb. 2006, pp. 1377-81.

[15] Alkaline Manganese Dioxide Handbook and Application Manual, Energizer Battery Manufacturing Inc., St. Louis, MO, 2007.

[16] Duracell Alkaline-Manganese Dioxide, Duracell Global OEM, Bethel, CT, 2006.

[17] MSP430 Flash Memory Characteristics (Application Report), Texas Instruments Inc., Dallas, TX, 2006.

[18] G. V. Merrett, A. S. Weddell, N. R. Harris, N. M. White, and B. M. AlHashimi, "The unified framework for sensor networks: A systems approach," unpublished, University of Southampton, Technical Report, Sept. 2006, http://eprints.ecs.soton.ac.uk/12955 